\title{
Two Novel Mutations of the NPM1 Gene in Syrian Adult Patients with Acute Myeloid Leukemia and Normal Karyotype
}

\author{
Ismael F. Alarbeed ${ }^{1 *}$, Abdulsamad Wafa ${ }^{2}$, Faten Moassass ${ }^{2}$, Balssel Al-Halabi², \\ Walid Alachkar ${ }^{2}$, Imad Aboukhamis ${ }^{1}$
}

\begin{abstract}
Objective: Somatic mutations in exon 12 of the NPM1 gene is one of the most common genetic abnormalities in adult acute myeloid leukemia (AML), which is observed in 25-35\% of AML patients and in 50-60\% of patients with cytogenetically normal AML (CN-AML). Methods: We performed Sanger sequencing of exon 12 of the NPM1 gene, on 44 CN-AML patients to characterize NPM1 status. Results: In this study, NPM1 mutations were identified in 10 $(22.7 \%)$ of the $44 \mathrm{CN}$-AML patients. Among the 10 patients with NPM1 mutations, type A NPM1 mutations were identified in $8(80 \%)$ patients, whereas non-A type NPM1 mutations were observed in $2(20 \%)$ patients. Two non-A type NPM1 mutations were not previously reported: c.867-868InsCGGA and c.861-862InsTGCA. These two novel mutant proteins display a nuclear export signal (NES) motif (L-xxx-L-xx-V-x-L) less frequently and L-x-Lx-V-xx-V$\mathrm{x}-\mathrm{L}$ it has been never seen before, yet. However, both novel mutations show a tryptophan loss at codon 288 and 290 at the mutant C-terminus which are crucial for aberrant nuclear export of NPM into the cytoplasm. Conclusions: This study suggests previously unreported NPM1 mutations may be non-rare and thus additional sequence analysis is needed along with conventional targeted mutational analysis to detect non type-A NPM1 mutations.
\end{abstract}

Keywords: Acute myeloid leukemia (AML)- normal karyotype- NPM1 gene mutation- prognositic factor

Asian Pac J Cancer Prev, 22 (1), 227-232

\section{Introduction}

Acute myeloid leukemia (AML) is a heterogeneous hematologic malignancy, which is characterized by uncontrolled proliferation of hematopoietic stem cells resulting in abnormal accumulation of myeloblasts. According to 2016 World Health Organization (WHO) classification AML has several cytogenetic and molecular subgroups (Arber et al., 2016). Cytogenetic and molecular findings help clinicians to stratify AML patients and plan therapeutic strategies. As far as cytogenetic abnormalities are concerned, the prognosis of AML patients was categorized into three risk groups: good, intermediate, and poor (Grimwade et al., 1998). However, approximately $50 \%$ of AML patients show up with a cytogenetically normal $(\mathrm{CN})$, representing a diverse subset of patients which are usually classified into an intermediate risk group (Gregory et al., 2009).

Nucleophosphomin (NPM1) is a nucleocytoplasmic shuttling protein that plays an active role in ribosomal protein assembly, chromatin remodeling, DNA repair, replication, and transcription (Lindstrom 2011). Mutations in the NPM1 gene have been reported in $25-35 \%$ of adult AML cases (Verhaak et al., 2005), which is a higher frequency $50-60 \%$ in CN-AML patients (Falini et al., 2005). However, more than 50 different mutations involving exon 12 of the NPM1 gene have been identified in AML. All of them lead to frameshift and elongation of the protein, which is aberrantly retained in cytoplasm (Falini et al., 2005). The presence of NPM1 mutations predict good response to induction therapy and it was associated with favorable outcome, increased complete remission (CR) rates, event-free survival (EFS) and overall survival (OS) (Liu et al., 2014).

The most common mutation in NPM1 patients is type A, which duplicates a TCTG tetranucleotide in the reference sequence at 956-959 and it accounts for up to $80 \%$ of adult $A M L$ with $N P M 1$ mutations (Koh et al., 2009). Several studies have suggested that non- A type NPM1 mutations may function as prognostic factors for poor clinical outcomes (Koh et al., 2009; Park et al., 2012). Therefore, it may be important to identify and characterize NPM1 mutations at the nucleotide and amino acid levels.

In this study, we evaluated for the first mutational spectrum of NPM1 mutations in adult CN-AML Syrian patients newly diagnosed, which was directly sequenced.

${ }^{1}$ Department of Microbiology, Hematology and Immunology, Faculty of Pharmacy, Damascus University, Ministry of High Education, Damascus, Syria. ${ }^{2}$ Department of Molecular Biology and Biotechnology, Human Genetics Division, Atomic Energy Commission, Damascus, Syria.*For Correspondence: drismaelalarbeed@gmail.com 
The biological and clinical features and the prognostic significance were also assessed.

\section{Materials and Methods}

\section{Subjects}

A total of 77 patients newly diagnosed with de novo AML between February 2018 and April 2019 were included in the study. Patients without previous treatment were included in the study; patients with normal karyotype were selected for molecular analysis and patients with history of exposure to chemotherapy/ radiotherapy, and secondary AML patients, were excluded. AML diagnosis was made according to French-AmericanBritish (FAB) classification. Their initial bone marrow $(\mathrm{BM})$ or peripheral blood (PB) samples were collected for use in the study. Patients consisted of 41 men and 36 women; the median age was $35.2 \pm 12.4$ years (range, 18-77 years) (Table 1). This study was approved by the Ethics Committee in Syrian Ministry of High Education and written informed consent was obtained from all the participants.

\section{Treatment Protocol}

All patients received $(3+7)$ standard induction chemotherapy, which consisted of daunorubicin at 45 $\mathrm{mg} / \mathrm{m} 2$ for 3 days and cytarabine at $100-200 \mathrm{mg} / \mathrm{m}^{2}$ for 7 days, followed by high doses of a cytarabine-based consolidation phase (cytarabine at $\mathrm{mg} / \mathrm{m}^{2} 3$ every $12 \mathrm{~h}$ for 3 days, repeated for 2 to 3 cycles). Patients with acute promyelocytic leukemia (M3) received all-trans retinoic acid plus anthracycline. Patients received conventional induction chemotherapy and were followed for $7.4 \pm 3.7$ months. BM aspiration was performed between 21 and 28 days after initiation of chemotherapy. The patients were followed up once every 3 months with clinical examination and complete blood counts. A BM aspiration was performed if there was any suggestion of relapse on clinical examination or PB smear.

\section{Cytogenetic and molecular cytogenetic analyses}

Chromosome analysis using GTG-banding was performed on BM sample prior to chemotherapy according to standard protocols (Alachkar et al., 2007). A normal male karyotype was diagnosed. Fluorescence in situ hybridization (FISH) using specific probes to detect translocations $\mathrm{t}(8 ; 21), \mathrm{t}(15 ; 17), \mathrm{t}(16 ; 16), \mathrm{t}(12 ; 21)$, and deletion del(13q), were performed with standard method to excluded patients with chromosomal abnormalities, as previously reported (Alachkar et al., 2007).

\section{Analysis of the NPM1 exon 12 mutations}

Genomic DNA was isolated from PB or BM samples from all 44 de novo AML patients using the QIAamp DNA Blood Mini kit (Qiagen, Germany) according to the manufactures instructions and was stored at $-20^{\circ} \mathrm{C}$. The exon 12 of the $N P M 1$ gene was amplified using specific primer NPM1-F 5'-TTAACTCTCTGGTGGTAGAATGAA-3' and NPM1-R 5'-CAAGACTATTTGCCATTCCTAAC-3'. The PCR reaction was performed in a total volume of $50 \mu \mathrm{l}$ containing 200 ng of genomic DNA, 10x PCR buffer (100
$\mathrm{mM}$ Tris- $\mathrm{HCl}, \mathrm{pH} 8.8,500 \mathrm{mM} \mathrm{KCl}), 2 \mathrm{mM} \mathrm{MgCl}, 200$ $\mu \mathrm{M}$ dNTPs, $10 \mathrm{pM}$ of each primer, and $1 \mathrm{U}$ of Taq DNA polymerase. PCR conditions included initial denaturation at $95^{\circ} \mathrm{C}$ for $5 \mathrm{~min}$ followed by 40 cycles of $94^{\circ} \mathrm{C}$ for 30 s, $57^{\circ} \mathrm{C}$ for $60 \mathrm{~s}$, and $72^{\circ} \mathrm{C}$ for $75 \mathrm{~s}$ with final extension at $72^{\circ} \mathrm{C}$ for $5 \mathrm{~min}$. The PCR products were purified and directly sequenced with reverse primer NPM1-R2 5'-GGCATTTTGGACAACACA-3' using the ABI Prism 310 genetic analyzer (Applied Biosystems, Foster City, CA, USA). The cycle-sequencing reaction was performed in a $10-\mu \mathrm{l}$ volume containing $1 \mu \mathrm{l}$ of the terminator ready reaction, $5 \mathrm{pmol}$ of either the forward or reverse primer and $10 \mathrm{ng}$ of purified PCR product (ExoSAP-IT kit; Amersham BioSciences, Piscataway, NJ, USA). The thermal cycle protocol was $95^{\circ} \mathrm{C}$ for 4 min followed by 30 cycles at $96^{\circ} \mathrm{C}$ for $10 \mathrm{sec}, 50^{\circ} \mathrm{C}$ for $5 \mathrm{sec}$ and $60^{\circ} \mathrm{C}$ for $4 \mathrm{~min}(\mathrm{ABI}$ GeneAmp PCR System 9700, Applied Biosystems). Centri-Sep columns (Princeton Separations, Adelphia, NJ, USA) were used for the effective and reliable removal of excess dye terminators (DyeEx 2.0, Qiagen, Germany) from completed DNA sequencing reactions. Data were compared and aligned with different sequences using the NCBI BLAST Assembled Genomes tool (http://blast.ncbi. nlm.nih.gov/Blast.cgi).

\section{Statistical Analysis}

The comparison of qualitative data such as age, WBC count, platelet count, hemoglobin level and blast count percentage between NPM1wt and NPM1mut patients were statistically evaluated using Fisher exact and chi-square tests. Overall survival (OS) was estimated for patients who received at least one induction course of therapy using the Kaplan-Meier method. P values $<0.05$ were considered significant. All analyses were performed using SPSS Statistics 19 software (SPSS, Chicago, IL, USA).

\section{Results}

A total of 77 newly diagnosed adult AML patients, the median age at diagnosis was $35.2 \pm 12.4$ years $(18-77$ years) were included in this study. Forty-four patients $(57.1 \%)$ showed a normal karyotype (NK) and 33 patients had chromosomal aberrations (42.9\%). NPM1 gene mutations were studied only in AML patients with NK. However, NPM1 gene mutations were identified in 10 of the 44 CN-AML patients $(22.7 \%)$ (Table 2). Regarding FAB subtype of $A M L, N P M 1$ mutations were found more frequently in M4 and/or M5 (80\%) than in the other subtypes of AML $(\mathrm{P}=0.04)$. Furthermore, the incidence of NPM1mut was significantly higher in older patients than young $(40.7$ vs. 33.6 years, $\mathrm{P}=0.1)$. NPM1 mutations were found more frequently in male $(60 \%)$ than female patients $(40 \%)$, but this difference was not significant $(\mathrm{P}=0.6)$.

Of the mentioned 10 patients, type A mutation (NM-002520.5) (c.860-863dupTCTG) was identified in eight patients $(80 \%)$, and type $Q$ mutation consisting of a 4-bp insertion between positions nt 964 and nt 965 (NM-002520), was identified in two patients (20\%): c.867-868InsCGGA (patient 16) and c.861-862InsTGCA (patient 21) (Figure 1). The predicted amino acid sequences were 287-LCLAVEEVSLRKX (patient 16) and 
A

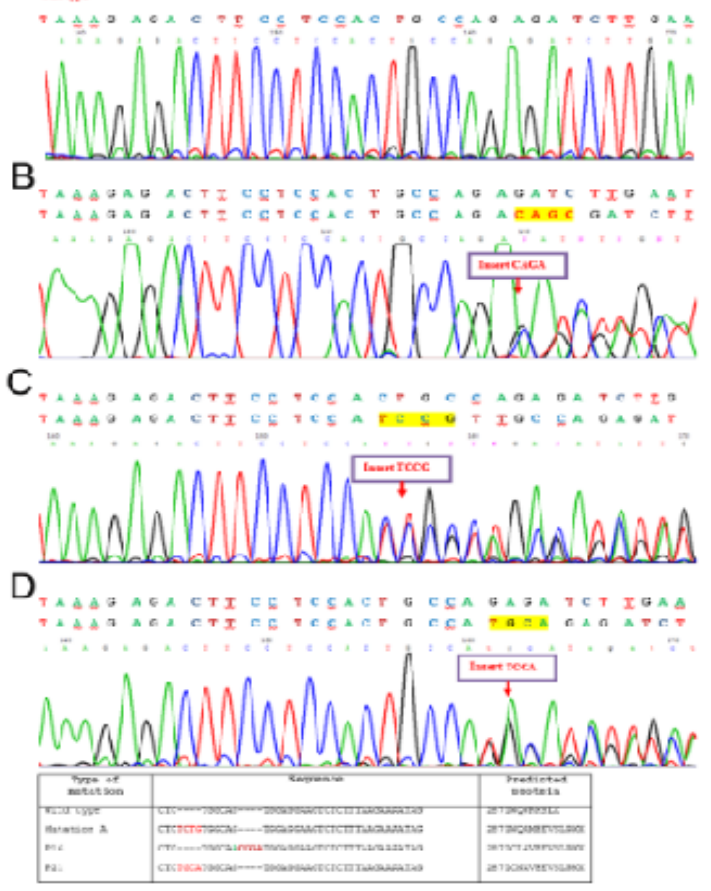

Figure 1. Nucleotide and Amino Acid Sequences of the Two Novel NPM1 Mutations. (A) Wild type of NPM1 sequence, (B) NPM1 mutation type A (c.860-863dupTCTG), (C) c.867-868InsCGGA (revers) (D) c.861-862InsTGCA (revers).

LCNAVEEVSLRKX (patient 21), respectively, instead of wide-type sequence 287-LWQWRKSLX (Figure 1).

However, there were no significant differences between NPM1 mut and NPM1 wt patients with respect to age, sex, hemoglobin, platelet counts and percentage of bone marrow blasts (Table 2). However, the mean WBC count at presentation in NPM1mut was significantly higher than NPM1wt patients (76.2 vs. $31.7 ; \mathrm{p}=0.04$ ).

Of all 44 patients who received standard induction chemotherapy, 39 patients $(88.6 \%)$ achieved CR. The CR rate was higher in patients with NPM1mut than NPM1 wt but the difference was not significantly $(\mathrm{p}=0.9)$. Moreover, of all patients who achieved CR, patients with NPM1mut had a higher relapse rate and a long survival than patients with NPM1 1 wt (relapse rate: $30 \%$ vs. $17.7 \%$, $p=0.08$; OS rate: 7.3 vs. 6.9 months, $p=0.09$ ). However, these differences were not statistically significant. NPM1mut were not independently associated with EFS or OS (Figure 2). In addition, a better median OS was observed in NPM1mt type-A compered NPM1mt non type-A patients when treated with intensive chemotherapy $(9,9$ months vs. did not reach the median; $\mathrm{p}=0.00)$ (data not shown).

\section{Discussion}

We report here for the first time two novel NPM1 mutations and frequency, distribution pattern and clinical impact in 44 adult Syrian patients with newly diagnosed CN-AML.

The frequency of NPM1 mutation in Asian AML series
OS

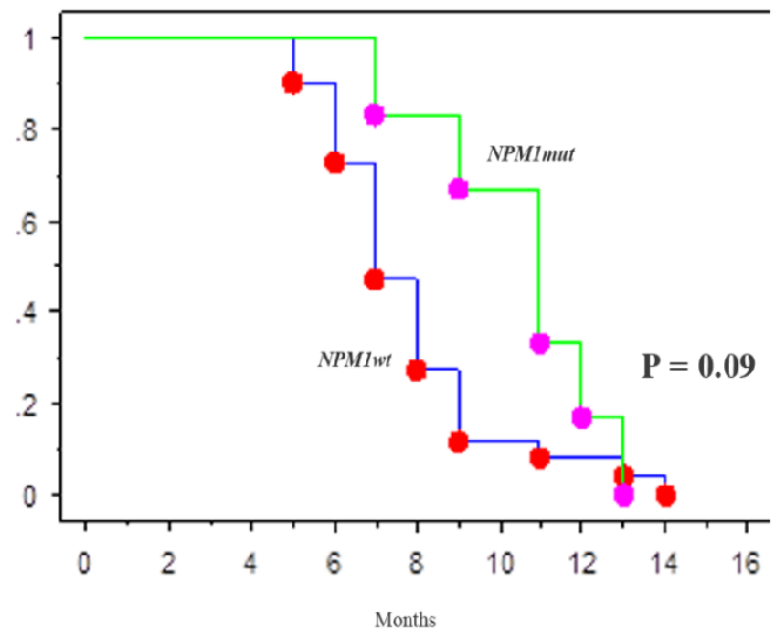

Figure 2. Kaplan-Meier Overall Survival Curves. OS of patients with CN-AML according to NPM1mut and NPMlwt.

Table 1. Demographic and Laboratory Data of A Do Novo Syrian Acute Myeloid Leukemia Patients

\begin{tabular}{|c|c|}
\hline Parameters & Value \\
\hline \multicolumn{2}{|l|}{ Gender } \\
\hline Male & $41(53.2 \%)$ \\
\hline Female & $36(46.8 \%)$ \\
\hline Sex ratio $(M / F)$ & 1.2 \\
\hline Age (median, range) & $35.2 \pm 12.4$ \\
\hline \multicolumn{2}{|l|}{ FAB classification } \\
\hline M0 & $1(1.3 \%)$ \\
\hline M1 & $10(13 \%)$ \\
\hline M2 & $13(16.9 \%)$ \\
\hline M3 & $17(22 \%)$ \\
\hline M4 & $25(32.5 \%)$ \\
\hline M5 & $10(13 \%)$ \\
\hline M6 & $1(1.3 \%)$ \\
\hline WBC, x $10^{9} / 1$ (median, range) & $44.02(0.8-300)$ \\
\hline $\mathrm{Hb}, \mathrm{g} / \mathrm{dl}$ (median, range) & $8.7(3.5-16.7)$ \\
\hline Plt, x 10¹/ (median, range) & $82.4(16-309)$ \\
\hline BM Blasts, $\%$ & $70(42-94)$ \\
\hline \multicolumn{2}{|l|}{ Cytogenetic status } \\
\hline Normal karyotype & $44(57.1 \%)$ \\
\hline Abnormal karyotype & $33(42.9 \%)$ \\
\hline \multicolumn{2}{|l|}{ Cytogenetic risk } \\
\hline Favorable & $11(14.3 \%)$ \\
\hline Intermediate & $59(76.6 \%)$ \\
\hline Adverse & $7(9 \%)$ \\
\hline \multicolumn{2}{|l|}{ NPM1 gene mutation status } \\
\hline Normal NPMI wide type & $34(77.3 \%)$ \\
\hline Mutant NPMI & $10(22.7)$ \\
\hline
\end{tabular}

FAB, French-American-British classifications; WBC, White blood cells; Hb, hemoglobin; Plt, Platelets; BM, bone marrow; CR, complete remission 
Table 2. Clinical Patients Characteristics According to NPM1 Status in Syrian AML Cytogeneticlly Normal Patients

\begin{tabular}{|c|c|c|c|}
\hline Features & $\begin{array}{c}\text { NPMI } \\
\text { Mutant group }\end{array}$ & $\begin{array}{l}N P M 1 \text { wide- } \\
\text { type group }\end{array}$ & $\mathrm{P}$ value \\
\hline Patients no $(\%)$ & $10(22.7 \%)$ & $34(77.3 \%)$ & \\
\hline \multicolumn{4}{|l|}{ Gender } \\
\hline Male & $6(60 \%)$ & $19(55.9 \%)$ & 0.6 \\
\hline Female & $4(40 \%)$ & $15(44.1 \%)$ & \\
\hline Sex ratio $(\mathrm{M} / \mathrm{F})$ & 1.5 & 1.2 & \\
\hline \multicolumn{4}{|l|}{ Age (years) } \\
\hline Mean & $40.7 \pm 13.3$ & $33.6 \pm 11.8$ & 0.1 \\
\hline range & $18-64$ & $18-57$ & \\
\hline \multicolumn{4}{|l|}{ WBC, x $10^{9} / 1$} \\
\hline Median & $76.2 \pm 97.1$ & $31.7 \pm 29.8$ & 0.04 \\
\hline Range & $7.9-300$ & $0.8-150$ & \\
\hline \multicolumn{4}{|l|}{$\mathrm{Hb}(\mathrm{g} / \mathrm{dl})$} \\
\hline Median & $8.8 \pm 1.5$ & $8.6 \pm 2.7$ & 0.9 \\
\hline Range & $6-10.8$ & $3.5-16.7$ & \\
\hline \multicolumn{4}{|l|}{ Plt x $10^{9} / 1$} \\
\hline Median & $75.6 \pm 27.4$ & $82.4 \pm 59.1$ & 0.8 \\
\hline Range & $37-110$ & $17-309$ & \\
\hline \multicolumn{4}{|l|}{ BM Blasts, $\%$} \\
\hline Median & $72.2 \pm 12$ & $68.5 \pm 15$ & 0.2 \\
\hline Range & $60-94$ & $42-90$ & \\
\hline FAB:M4\&M5/others & $8(80 \%)$ & $15(44.1 \%)$ & 0.04 \\
\hline
\end{tabular}

FAB, French-American-British classifications; WBC, White blood cells; $\mathrm{Hb}$, hemoglobin; Plt, Platelets; BM, bone marrow; CR, complete remission; $\mathrm{P}<0.05$ is considered significant

varied between 20-32\% (Suzuki et al., 2005; Chou et al., 2006; Yan et al., 2007a; Yan et al., 2007b; Boonthimat et al., 2008; Ruan et al., 2009). Whereas, it accounts in adult AML-patients of other populations between 21 and 25\% (Roti et al., 2006; Lin et al., 2006; Mori et al., 2007; Ahmad et al., 2009), while reports from Thailand, China and the most European countries were between 26 and 35\% (Falini et al., 2005; Yan et al., 2007a; Yan et al., 2007b;Boonthimat et al., 2008). However, in this study incidence of NPM1 mutation in adult AML patients was $22.7 \%$; our findings were closer to observations from India (21\%) (Chauhan et al., 2013), Egypt (21.8\%) (Zidan et al., 2013), and Iran (20.8\%) (Rezaei et al., 2017) and in agreement with other previous studies (Roti et al., 2006; Lin et al., 2006; Mori et al., 2007; Ahmad et al., 2009). The differences in those results may be explained due to sample sizes as well as geographic and ethnic background of the studied populations.

Furthermore, the current study is in agreement with previous reports, which suggested that patients had higher WBC count in mutant versus wild type group $(\mathrm{P}=0.04)$ (Chou et al., 2006; Döhner et al., 2005) but no significant differences with regard to hemoglobin level and platelets count (Lin et al., 2006 ); high incidence of mutation in AML-M4/M5 subtype ( $\mathrm{P}=0.04)$ was also observed (Chou et al., 2006; Schnittger et al., 2005; Garzon et al., 2008).

Regardless of the NPM1 mutations type, type A mutation is the most common change, it accounts in up to $80 \%$ of adult AML patients and it has the NES motif L-xxx-V-xx-V-x-L (Koh et al., 2009). The mutations are characterized by frameshift insertions in the region encoding the $\mathrm{C}$-terminus of the protein, leading to the disruption of tryptophan residues 288 and 290 and the generation of an additional NES motif, which ultimately leads to the cytoplasmic localization of the NPM1-mut as well as NPM1-wt proteins (Falini et al., 2005; Falini et al., 2006; Mariano et al., 2006). However, NPM1 mut encodes cytoplasmic NPM1, which acts as an oncoprotein (Chou et al., 2006). Interestingly, we identified two novel NPM1 mutations of type Q never been reported before yet (according to COSMIC database for somatic samples from hematopoietic and lymphoid tissue), both novel NPM1 mutations that were identified in this study have a rare NES motif L-xxx-L-xx-V-x-L (Falini et al., 2006; Mariano et al., 2006) and L-x-Lx-V-xx-V-x-L it has been never seen before, yet. The common NES motif requires the loss of both tryptophans 288 and 290 to be transported out of the nucleus efficiently (Falini et al., 2006). However, both novel mutations in our study show a tryptophan loss at codon 288 and 290 at the mutant C-terminus which are crucial for aberrant nuclear export of NPM into the cytoplasm.

Two studies suggested that a type A mutant impacted patient prognosis favorably (Garzon et al., 2008; Hollink et al., 2009). Conflicting findings have been reported in the relationship between NPM1 non-A mutations and prognostic (Chou et al., 2006; Ahmad et al., 2009; Ahmad et al., 2010; Pastore et al., 2014; Hollink et al., 2009). Several studies did not demonstrate any differences in outcomes between the different types of NPM1 mutations in pediatric AML (Ahmad et al., 2010; Pastore et al., 2014; Hollink et al., 2009). Whereas, some authors showed that patients with non-A mutations have an adverse impact on survival (Chou et al., 2006; Ahmad et al., 2009). Recent study reported that high variant allele frequency of NPM1 predict poor outcomes in de novo AML; and this effect was not affected by FLT/ITD (Patel et al., 2019).

Regarding to the difference between NPM1 mut type-A and NPM1mut non- type-A patients with achievement of $\mathrm{CR}$ and OS. However, no significant differences between NPM1mut type-A and NPM1mut non type-A patients was reported with regard to $\mathrm{CR}$ and OS (Balatzenko et al., 2014; Boissel et al., 2005). On the other hand, non-A-type NPM1mut patients were associated with a significantly shorter CR rate and shorter OS compared with NPM1wt and A-type NPM1mut (Ahmad et al., 2009). We noted similar findings as reported in (Ahmad et al., 2009), patients with NPM1mut type-A had a better OS than NPM1mut non type-A (9.9 months vs. did not reach the median; $p=0.00$ ); still, our results need to be confirmed on larger numbers of patients for each group and need to be studied with other potential co-occurring genes affected, such as FLT3-ITD.

In conclusions, we report here for the first time two novel NPM1 mutations that were identified in adult Syrian CN-AML patients. Although the prognostic value of these non-A types of NPM1 mutations requires 
further investigation, the incidence of NPM1 mutations in adult Syrian AML patients was similar to that reported by other studies. High WBC, dominant M4 and/or M5 subtypes, a better OS and CR characterized NPM1mut patients in our study. Molecular assessment of NPMI mutation at diagnosis offers valuable additional prognostic information and may there by markedly affect therapeutic decisions.

\section{Acknowledgements}

We express gratitude to our Research Team, Faculty of Pharmacy of Damascus University and Atomic Energy Commission of SYRIA (AECS) for their support.

\section{Conflict of Interest}

The authors declare that they have no competing interests.

\section{References}

Ahmad F, Mandava S, Das BR (2009). Mutations of NPM1 gene in de novo acute myeloid leukaemia: determination of incidence, distribution pattern and identification of two novel mutations in Indian population. Hematol Oncol, 27, 90-97.

Ahmad F, Mandava S, Das BR (2010). Analysis of FLT3-ITD and FLT3-Asp835 mutations in de novo acute myeloid leukemia: evaluation of incidence, distribution pattern, correlation with cytogenetics and characterization of internal tandem duplication from Indian population. Cancer Invest, 28, 63-73.

Alachkar W, Wafa A, Nweder MS (2007). A complex translocation $\mathrm{t}(5 ; 9 ; 22)$ in Philadelphia cells involving the short arm of chromosome 5 in a case of chronic myelogenous leukemia. J Exp Clin Cancer Res, 26, 411-5.

Arber DA, Orazi A, Hasserjian R, et al (2016). The 2016 revision to the World Health Organization classification of myeloid neoplasms and acute leukemia. Blood, 127, 2391-2405.

Balatzenko G, Spassov B, Stoyanov N, et al (2014). NPM1 gene type A mutation in Bulgarian adults with acute myeloid leukemia: A Single-Institution Study NPM1 Gene Type A Mutation in Bulgarian Adults with Acute Myeloid Leukemia: A Single-Institution Study. Turk J Haematol, 31, 40-48.

Boissel N, Renneville A, Biggio V, et al (2005). Prevalence, clinical profile, and prognosis of NPM mutations in AML with normal karyotype. Blood, 106, 3618-20.

Boonthimat C, Thongnoppakhun W, Auewarakul CU (2008). Nucleophosmin mutation in Southeast Asian acute myeloid leukemia: eight novel variants, FLT3 coexistence and prognostic impact of NPM1/FLT3 mutations. Haematologica, 93, 1565-9.

Chauhan PS, Ihsan R, Singh LC, et al (2013). Mutation of NPM1 and FLT3 genes in acute myeloid leukemia and their association with clinical and immunophenotypic features. Dis Mark, 35, 581-8.

Chou WC, Tang JL, Lin LI, et al (2006). Nucleophosmin mutations in de novo acute myeloid leukemia: the agedependent incidences and the stability during disease evolution. Cancer Res, 66, 3310-6.

Döhner K, Schlenk RF, Habdank M, et al (2005). Mutant nucleophosmin (NPM1) predicts favorable prognosis in younger adults with acute myeloid leukemia and normal cytogenetics: interaction with other gene mutations. Blood, 106, 3740-6.

Falini B, Mecucci C, Tiacci E, et al (2005). Cytolasmatic nucleophosmin in acute myelogenous leukemia with normal karyotype. N Engl J Med, 352, 254-66.

Falini B, Bolli N, Shan J, et al (2006). Both carboxy-terminus NES motif and mutated tryptophan(s) are crucial for aberrant nuclear export of nucleophosmin leukemic mutants in NPMc+ AML. Blood, 107, 4514-23.

Garzon R, Garofalo M, Martelli MP, et al (2008). Distinctive microRNA signature of acute myeloid leukemia bearing cytoplasmic mutated nucleophosmin. PNAS, 105, 3945-50.

Gregory TK, Wald D, Chen Y, et al (2009). Molecular prognostic markers for adult acute myeloid leukemia with normal cytogenetics. J Hematol Oncol, 2, 23.

Grimwade D, Walker H, Oliver F, et al (1998). The importance of diagnostic cytogenetics on outcome in AML: analysis of 1,612 patients entered into the MRC AML 10 trial. Blood, 92, 2322-33.

Hollink I, Zwaan CM, Zimmermann M, et al (2009). Favorable prognostic impact of NPM1 gene mutations in childhood acute myeloid leukemia, with emphasis on cytogenetically normal AML. Leukemia, 23, 262-70.

Koh Y, Park J, Bae EK, et al (2009). Non-A type nucleophosmin 1 gene mutation predicts poor clinical outcome in de novo adult acute myeloid leukemia: differential clinical importance of NPM1 mutation according to subtype. Int $J$ Hematol, 90, 1-5.

Lin LI, Lin TC, Chou WC, et al (2006). A novel fluorescencebased multiplex PCR assay for rapid simultaneous detection of CEBPA mutations and NPM mutations in patients with acute myeloid leukemias. Leukemia, 20, 1899-1903.

Lindstrom MS (2011). NPM1/B23. A multifunctional chaperone in ribosome biogenesis and chromatin remodeling. Biochem Res Int, 2011, 195209.

Liu Y, He P, Liu F, et al (2014). Prognostic significance of NPM1 mutations in acute myeloid leukemia: A meta-analysis. $\mathrm{Mol}$ Clin Oncol, 2, 275-81.

Mariano AR, Colombo E, Luzi L, et al (2006). Cytoplasmic localization of NPM in myeloid leukemias is dictated by gain-of-function mutations that create a functional nuclear export signal. Oncogene, 25, 4376-80.

Mori Y, Yoshimoto G, Kumano T, et al (2007). Distinctive expression of myelomonocytic markers and down-regulation of CD34 in acute myelogenous leukaemia with FLT3 tandem duplication and nucleophosmin mutation. Eur J Hematol, 79, 17-24.

Park BG, Chi HS, Park SJ, et al (2012). Clinical implications of non-A-type NPM1 and FLT3 mutations in patients with normal karyotype acute myeloid leukemia. Acta Haematol, 127, 63-71.

Pastore F, Greif PA, Schneider S, et al (2014). The NPM1 mutation type has no impact on survival in cytogenetically normal AML. PLoS One, 9, e109759.

Patel SS, Pinkus GS, Ritterhouse LL, et al (2019). High NPM1 mutant allele burden at diagnosis correlates with minimal residual disease at first remission in de novo acute myeloid leukemia. Am J Hematol, 94, 921-8.

Rezaei N, Arandi N, Valibeigi B, et al (2017). FMS-Like Tyrosine Kinase 3 (FLT3) and Nucleophosmin 1 (NPM1) in Iranian adult acute myeloid leukemia patients with normal Karyotypes: Mutation Status and Clinical and Laboratory Characteristics. Turk J Haematol, 34, 300-306.

Roti G, Rosati R, Bonasso R, et al (2006). Denaturing highperformance liquid chromatography: a valid approach for identifying NPM1 mutations in acute myeloid leukemia. $J$ Mol Diagn, 8, 254-9.

Ruan GR, Li JL, Qin YZ, et al (2009). Nucleophosmin mutations in Chinese adults with acute myelogenous leukemia. Ann Hematol, 88, 159-66. 
Schnittger S, Schoch C, Kern W, et al (2005). Nucleophosmin gene mutations are predictors of favorable prognosis in acute myelogenous leukemia with a normal karyotype. Blood, 106, 3733-9.

Suzuki T, Kiyoi H, Ozeki K, et al (2005). Clinical characteristics and prognostic implications of NPM1 mutations in acute myeloid leukemia. Blood, 106, 2854-61.

Verhaak RG, Goudswaard CS, van Putten W, et al (2005). Mutations in nucleophosmin (NPM1) in acute myeloid leukemia (AML): association with other gene abnormalities and previously established gene expression signatures and their favorable prognostic significance. Blood, 106, 3747-54.

Yan L, Chen S, Liang J, et al (2007a). Analysis of NPM1 gene mutations in Chinese adults with acute myeloid leukemia. Int J Hematol, 86, 143-6.

Yan LZ, Chen SN, Liang JY, et al (2007b). Analysis of NPM1 gene mutations in acute myeloid leukaemia. Zhonghua Xue Ye Xue Za Zhi, 28, 289-93.

Zidan M, Shaaban H, Ghannam DE (2013). Prognostic impact of nucleophosmin 1 (NPM1) gene mutations in Egyptian acute myeloid leukemia patients. Turk J Haematol, 30, 129-36.

This work is licensed under a Creative Commons AttributionNon Commercial 4.0 International License. 